\title{
REVIEW
}

\section{Role of beneficial elements in plants: implications for the photosynthetic process}

\author{
E. LO PICCOLO*, C. CECCANTI*, L. GUIDI ${ }^{*, * *,}$, and M. LANDI ${ }^{*, * *}$
}

Department of Agriculture, Food and Environment, University of Pisa, Via del Borghetto 80, 56124 Pisa, Italy* CIRSEC, Centre for Climate Change Impact, University of Pisa, Via del Borghetto 80, 56124 Pisa, Italy ${ }^{* *}$

\begin{abstract}
Essential macro- and microelements availability strictly influences photosynthetic process as well as the overall plant status. Besides essential macro- and micronutrients, a group of other elements described as 'beneficial' has been proposed to ameliorate plant performances under optimal and/or stressful conditions. The first explanation of the term 'essential', as suggested by Arnon and Stout in 1939, defined beneficial elements as not essential since they are not necessary for plants to complete their life cycle. However, at low amounts, they can exert promoting effects on plant performances, including photosynthesis. In this review, we summarize the positive effect of seven beneficial elements ( $\mathrm{Se}, \mathrm{Si}, \mathrm{Na}, \mathrm{I}, \mathrm{Ti}, \mathrm{Ce}$, and $\mathrm{La}$ ) on plant photosynthetic processes to depict a clear picture of the influences of these beneficial elements on photosynthesis.
\end{abstract}

Keywords: ameliorative role; nonessential elements; nutrients; plant nutrition; stress condition.

\section{Introduction}

The term 'essential element' for plant nutrition was proposed by Arnon and Stout (1939), and successively modified by Epstein (1965), as an element necessary to complete the plant life cycle. However, to date, essential elements are still an open question remaining an intricate and dynamic research topic (Marschner 2012). If macroelements (Ca, K, Mg, N, S, and P) are well defined, being usually present in plant tissues at concentrations $>1,000$ $\mathrm{mg} \mathrm{kg}^{-1}$ (dry mass, DM), in contrast, micronutrients remain an open topic, due to their lower concentrations in tissues [usually $\leq 100 \mathrm{mg} \mathrm{kg}^{-1}(\mathrm{DM})$ ]. There is a fine line between the microelements and 'beneficial' elements. The latter are not necessary to a plant life cycle but they can improve plant performances under optimal or stressful conditions (Vatansever et al. 2017). This has led several authors to consider some microelements as essential or beneficial (Broadley et al. 2012, Vatansever et al. 2017). So, no wonder that some controversial element will be included/ excluded in the list of essential plant microelements in the future through further molecular and physiological studies.

Photosynthesis is the process that fuels our biosphere and some microelements, such as $\mathrm{Cu}, \mathrm{Fe}$, and $\mathrm{Mn}$, are necessary for the correct functioning of photosynthetic process as they are constituents or cofactors of many

\section{Highlights}

- Beneficial elements (BE) have been proposed to ameliorate plant performances

- Se, Si, Na, I, Ti, Ce, and La were reported to improve photosynthetic traits

- Bases of photosynthesis improvement by BE are far from being fully elucidated
Received 21 April 2021

Accepted 16 June 2021

Published online 25 June 2021

Corresponding author phone: +39050 2216613

e-mail: lucia.guidi@unipi.it

Abbreviations: Car - carotenoids; Chl - chlorophylls; $C_{\mathrm{i}}-$ intercellular $\mathrm{CO}_{2}$ concentration; Cyt $b_{6} / f-$ cytochrome $b_{6} f$ complex; $E$ - transpiration rate; ETR - electron transport rate; $\mathrm{F}_{\mathrm{m}}$ - maximum chlorophyll fluorescence yield; $\mathrm{F}_{\mathrm{v}} / \mathrm{F}_{\mathrm{m}}-\mathrm{maximum}_{\mathrm{m}}$ photochemical efficiency of PSII; $g_{\mathrm{m}}-$ mesophyll conductance; $g_{\mathrm{s}}-$ stomatal conductance; $J_{\max }-$ maximum rate of electron transport; $\mathrm{NP}_{\mathrm{S}}-$ nanoparticles; OEC - oxygen-evolving complex; $P_{\mathrm{N}}$ - net assimilation of photosynthesis; $\mathrm{q}_{\mathrm{P}}$ - photochemical quenching coefficient; ROS - reactive oxygen species; $V_{\text {cmax }}$ - maximum carboxylation rate; WUE - water-use efficiency; $\Phi_{\text {PSII }}-$ effective quantum yield of PSII.

Conflict of interest: The authors declare that they have no conflict of interest. 
proteins and enzymes (Aggarwal et al. 2012). However, also elements called 'beneficial', administered at low doses in plants, can effectively stimulate the photosynthetic activity, probably due to nonspecific reactions in plants leading to the eustress phenomenon (Nyitrai et al. 2003). Moreover, beneficial elements, such as $\mathrm{Se}, \mathrm{Si}, \mathrm{Na}, \mathrm{I}, \mathrm{Ti}, \mathrm{Ce}$, and La, can additionally exert peculiar features, becoming constituents of cellular structures and proteins involved in photosynthesis or having photo-electromagnetic effects on photosynthesis (Brownell and Bielig 1996, Hong et al. 2002, Yamamoto et al. 2012, Andrade et al. 2018, Kiferle et al. 2021).

Previous literature surveys described the role of these beneficial elements in planta (Pilon-Smits et al. 2009, Broadley et al. 2012, Vatansever et al. 2017, Lyu et al. 2017, Gómez-Merino and Trejo-Téllez 2018) while this review aims to describe the specific effects of $\mathrm{Se}, \mathrm{Si}, \mathrm{Na}$, I, Ti, Ce, and La on plant photosynthetic traits under both optimal and stressful conditions. However, we are aware that from the works presented in this review, the influence of a specific beneficial element on photosynthesis could both be ascribable to the ion form and also to the plant species. In addition, for certain particular plant taxa, some of these elements are essential (e.g., sodium for halophytes), which, conversely, might become beneficial for plants belonging to most of the other taxa. This review attempts to provide a starting point to stimulate further molecular and physiological studies to clarify the promoting effects of these beneficial elements to the photosynthetic process.

\section{Selenium}

Selenium is one of the most studied beneficial elements in plants and often used in plant biofortification programs, due to its positive effects on the human health (Malagoli et al. 2015). Selenate and selenite are the two major chemical forms of Se bioavailable in the soil. Selenate is transported in plants through sulphate transporters since both ions showed molecular similarity, while selenite is taken up by phosphate transporters (White 2018, Trippe and Pilon-Smits 2021).

In plants, Se plays a positive role in photosynthesis, chloroplast and cell membrane development, and it is also involved in antioxidant and ROS regulation (PilonSmits 2015). Indeed, Se can improve the growth and plant performance at lower concentrations reducing the possible detrimental effects of abiotic stressors (Amirabad et al. 2020).

In plants subjected to drought stress, it was reported that Se, either added to the soil or sprayed on leaves, improved the photosynthetic rate (e.g., Germ et al. 2007, Andrade et al. 2018); however, a positive effect on gasexchange parameters was also observed during heat (Haghighi et al. 2019), salinity (Jiang et al. 2017), and Cd stresses (Filek et al. 2010, Amirabad et al. 2020). Under these stressful conditions, higher levels of $g_{\mathrm{s}}, E, P_{\mathrm{N}}$, WUE, and better maintenance of PSII performances (higher $\mathrm{F}_{\mathrm{v}} / \mathrm{F}_{\mathrm{m}}$ and $\left.\Phi_{\text {PSII }}\right)$ were observed with respect to control (see references in Table 1).
Similar effects of Se were observed in Triticum aestivum (Nawaz et al. 2015) and Olea europaea (Proietti et al. 2013) subjected to drought conditions. Under salinity, Habibi (2017a) found that higher $\mathrm{F}_{\mathrm{v}} / \mathrm{F}_{\mathrm{m}}$ values in Petroselinum crispum plants treated with $\mathrm{Se}$ as compared to the controls were attributable to lower damage to PSII.

The positive impact of Se on $g_{\mathrm{s}}, E, P_{\mathrm{N}}$, and WUE could be very useful for plants growing in arid, semiarid, or salty areas. This protective effect of Se on photosynthetic processes consisted in minimizing damages to the chloroplast structure and enhancing the electron transport rate (Andrade et al. 2018). Indeed, Djanaguiraman et al. (2010), reported that Se application in sorghum plants grown under high temperature stress conditions increased the antioxidant defence and, in this way, increased the photosynthetic rate and grain yield. Plants cultivated with Se have a strengthened antioxidative capacity due to increased antioxidant enzyme activity and decreased ROS content (Xue et al. 2001). This concept is reinforced by Alves et al. (2020) reporting that Se can alleviate the negative effects of Cd-stress conditions and also enhance the tolerance of tomato plants against this trace element by improving the nutritional status, the proline content, and increasing the activity of antioxidant enzymes.

It is evident that low concentrations of Se (e.g., $15 \mu \mathrm{M}$ $\mathrm{Na}_{2} \mathrm{SeO}_{3} \cdot 5 \mathrm{H}_{2} \mathrm{O}$ ) can enhance the activity of some antioxidant enzymes, such as ascorbate peroxidase, glutathione reductase, dehydroascorbate reductase, and monodehydroascorbate reductase in Zea mays (Yildiztugay et al. 2017), glutathione reductase and glutathione peroxidase in T. aestivum (Balakhnina and Nadezhkina 2017), and superoxide dismutase and glutathione peroxidase in Solanum lycopersicum (Castillo-Godina et al. 2016). Also, under heat stress, in Sorghum bicolor plants, Djanaguiraman et al. (2010) demonstrated that the ameliorative effect of Se on photosynthesis resulted from lower ROS accumulation in mesophyll achieved by enhancing antioxidant enzyme activities.

In conclusion, even though the exact way by which selenium promotes benefits in plants remains until unknown, certainly Se can restore the photosynthetic capacity, improve antioxidant system and enhance crop production in plants subjected to environmental stresses (Feng et al. 2013, Alyemeni et al. 2018).

\section{Silicon}

In the Earth's crust, Si represents the second most abundant element; therefore, it can be present at relevant concentrations in the rhizosphere (Broadley et al. 2012, Vatansever et al. 2017, Sheik et al. 2018). Among Si accumulator plants, monocotyledons (e.g., Poaceae) usually accounted for higher $\mathrm{Si}$ contents than that of dicotyledons (e.g., Cucurbitales and Urticales) (Raven 2003, Kaur et al. 2016). Silicon is transported in plants as $\mathrm{Si}(\mathrm{OH})_{4}$, then converted and stored to hydrated amorphous silica on cell walls (Vatansever et al. 2017). Silicon enhances the strength and the rigidity of cell wall due to a positive interaction with pectin and polyphenols (Zhang 
Table 1. Effect of selenium (Se) on plant photosynthetic traits.

\begin{tabular}{|c|c|c|c|c|c|c|c|}
\hline $\begin{array}{l}\text { Beneficial } \\
\text { element }\end{array}$ & $\begin{array}{l}\text { Administra- } \\
\text { tion method }\end{array}$ & $\begin{array}{l}\text { Chemical form and } \\
\text { concentration }\end{array}$ & Species & Stress type & Observation & $\begin{array}{l}\text { Concentration in } \\
\text { plant tissues }\end{array}$ & Reference \\
\hline \multirow[t]{18}{*}{ Selenium } & Leaf & $1 \mathrm{~g}\left(\mathrm{Na}_{2} \mathrm{SeO}_{4}\right) \mathrm{m}^{-3}$ & $\begin{array}{l}\text { Fagopyrum } \\
\text { esculentum } \\
\text { (Moench) cv. Darja } \\
\text { and Fagopyrum } \\
\text { tataricum (L.) } \\
\text { Gaertn. }\end{array}$ & UV-B & Higher $\Phi_{\mathrm{PSII}}$ & - & $\begin{array}{l}\text { Breznik et al. } \\
(2005)\end{array}$ \\
\hline & Leaf & $10 \mathrm{mg}\left(\mathrm{Na}_{2} \mathrm{SeO}_{4}\right) \mathrm{L}^{-1}$ & $\begin{array}{l}\text { Solanum } \\
\text { tuberosum L. }\end{array}$ & Drought & Higher $\Phi_{\mathrm{PSII}}$ & $\begin{array}{l}258-730 \mathrm{ng} \\
\mathrm{g}^{-1}(\text { leafDM })\end{array}$ & $\begin{array}{l}\text { Germ et al. } \\
(2007)\end{array}$ \\
\hline & Leaf & $1 \mathrm{~g}\left(\mathrm{Na}_{2} \mathrm{SeO}_{4}\right) \mathrm{m}^{-3}$ & $\begin{array}{l}\text { Fagopyrum } \\
\text { esculentum Moench. }\end{array}$ & Drought & $\begin{array}{l}\text { Higher } g_{\mathrm{s}} \text { and } \\
\Phi_{\text {PSII }}\end{array}$ & - & $\begin{array}{l}\text { Tadina et al. } \\
\text { (2007) }\end{array}$ \\
\hline & Leaf & $75 \mathrm{mg}\left(\mathrm{Na}_{2} \mathrm{SeO}_{4}\right) \mathrm{L}^{-1}$ & $\begin{array}{l}\text { Sorghum } \\
\text { bicolor (L.) Moench }\end{array}$ & Heat & $\begin{array}{l}\text { Higher } P_{\mathrm{N}}, g_{\mathrm{s}} \text {, } \\
\text { and } E\end{array}$ & $\begin{array}{l}20-30 \mu \mathrm{g} \\
\mathrm{g}^{-1}(\text { leaf DM })\end{array}$ & $\begin{array}{l}\text { Djanaguiraman } \\
\text { et al. }(2010)\end{array}$ \\
\hline & $\begin{array}{l}\text { Nutrient } \\
\text { solution }\end{array}$ & $2 \mu \mathrm{M} \mathrm{Na}_{2} \mathrm{SeO}_{4}$ & $\begin{array}{l}\text { Brassica } \\
\text { napus L. }\end{array}$ & Cadmium & $\begin{array}{l}\text { Higher } \mathrm{F}_{\mathrm{v}} / \mathrm{F}_{\mathrm{m}} \text {, } \\
\Phi_{\mathrm{PSII}} \text {, and } \mathrm{q}_{\mathrm{P}}\end{array}$ & - & $\begin{array}{l}\text { Filek et al. } \\
\text { (2010) }\end{array}$ \\
\hline & Leaf & $30 \mathrm{~g}\left(\mathrm{Na}_{2} \mathrm{SeO}_{4}\right) \mathrm{ha}^{-1}$ & $\begin{array}{l}\text { Hordeum } \\
\text { vulgare L. }\end{array}$ & Drought & $\begin{array}{l}\text { Higher } \mathrm{F}_{\mathrm{v}} / \mathrm{F}_{\mathrm{m}} \\
\text { and } g_{\mathrm{s}}\end{array}$ & - & $\begin{array}{l}\text { Habibi } \\
(2013)\end{array}$ \\
\hline & Leaf & $\begin{array}{l}50-150 \\
\mathrm{mg}\left(\mathrm{Na}_{2} \mathrm{SeO}_{4}\right) \mathrm{L}^{-1}\end{array}$ & $\begin{array}{l}\text { Olea } \\
\text { europaea L. }\end{array}$ & Drought & $\begin{array}{l}\text { Higher } E, P_{\mathrm{N}} \text {, } \\
\text { and } g_{\mathrm{s}}\end{array}$ & $\begin{array}{l}26-36 \mu \mathrm{g} \\
\mathrm{g}^{-1}(\text { leaf FM })\end{array}$ & $\begin{array}{l}\text { Proietti et al. } \\
\text { (2013) }\end{array}$ \\
\hline & Substrate & $\begin{array}{l}10,20,50 \\
\mathrm{~g}\left(\mathrm{Na}_{2} \mathrm{SeO}_{3}\right) \mathrm{ha}^{-1}\end{array}$ & $\begin{array}{l}\text { Oryza } \\
\text { sativa } \mathrm{L} .\end{array}$ & No stress & $\begin{array}{l}\text { Higher } P_{\mathrm{N}}, C_{\mathrm{i}}, \\
E, \mathrm{~F}_{\mathrm{v}}, \mathrm{F}_{\mathrm{m}}, \mathrm{F}_{\mathrm{v}} / \mathrm{F}_{\mathrm{m}}, \\
\text { and } \mathrm{F}_{\mathrm{v}} / \mathrm{F}_{0}\end{array}$ & $\begin{array}{l}0.9-5.5 \mathrm{mg} \\
\mathrm{kg}^{-1}(\mathrm{shoot} \mathrm{DM}), \\
0.2-1.2 \mathrm{mg} \\
\mathrm{kg}^{-1}(\text { root DM }) \\
40-70 \mu \mathrm{g} \\
\mathrm{kg}^{-1}(\text { grain DM })\end{array}$ & $\begin{array}{l}\text { Zhang et al. } \\
\text { (2014) }\end{array}$ \\
\hline & Leaf & $0.017 \mathrm{~g}(\mathrm{Se}) \mathrm{L}^{-1}$ & $\begin{array}{l}\text { Pyrus } \times \\
\text { bretschneideri } \\
\text { Rehder, Vitis } \\
\text { vinifera L., and } \\
\text { Prunus persica (L.) } \\
\text { Batsch }\end{array}$ & No stress & $\begin{array}{l}\text { Higher } P_{\mathrm{N}}, g_{\mathrm{s}} \text {, } \\
E \text {, and } C_{\mathrm{i}}\end{array}$ & $\begin{array}{l}0.0041 \mu \mathrm{g} \mathrm{g}^{-1}(\mathrm{FM}) \\
\text { in pear, } \\
0.0092 \mu \mathrm{g} \mathrm{g}^{-1}(\mathrm{FM}) \\
\text { in grape, } \\
0.0038 \mu \mathrm{g} \mathrm{g}^{-1}(\mathrm{FM}) \\
\text { in peach }\end{array}$ & $\begin{array}{l}\text { Feng et al. } \\
(2015)\end{array}$ \\
\hline & Substrate & $\begin{array}{l}3-24 \mathrm{mg}\left(\mathrm{Na}_{2} \mathrm{SeO}_{3}\right) \\
\mathrm{kg}^{-1}\end{array}$ & $\begin{array}{l}\text { Nicotiana } \\
\text { tabacum L. }\end{array}$ & No stress & $\begin{array}{l}\text { Higher } P_{\mathrm{N}}, g_{\mathrm{s}} \text {, } \\
\text { and } C_{\mathrm{i}}\end{array}$ & $\begin{array}{l}4.13-31.36 \mathrm{mg} \\
\mathrm{kg}^{-1} \text { (root DM), } \\
0.53-3.66 \mathrm{mg} \\
\mathrm{kg}^{-1}(\text { stem DM), } \\
1.17-7.52 \mathrm{mg} \\
\mathrm{kg}^{-1} \text { (leaf DM) }\end{array}$ & $\begin{array}{l}\text { Jiang et al. } \\
(2015)\end{array}$ \\
\hline & Leaf & $40 \mathrm{mg}\left(\mathrm{Na}_{2} \mathrm{SeO}_{4}\right) \mathrm{L}^{-1}$ & $\begin{array}{l}\text { Triticum } \\
\text { aestivum } \mathrm{L} .\end{array}$ & Drought & $\begin{array}{l}\text { Higher } E, P_{\mathrm{N}} \text {, } \\
\text { and } g_{\mathrm{s}}\end{array}$ & $2-6 \mu \mathrm{g} \mathrm{g}^{-1}$ (shoot) & $\begin{array}{l}\text { Nawaz et al. } \\
(2015)\end{array}$ \\
\hline & Substrate & $\begin{array}{l}20,40,80 \mu \mathrm{M} \\
\mathrm{Na}_{2} \mathrm{SeO}_{4}\end{array}$ & $\begin{array}{l}\text { Brassica } \\
\text { juncea } \mathrm{L} \text {. }\end{array}$ & $\begin{array}{l}\text { Epibrassi- } \\
\text { nolide }\end{array}$ & $\begin{array}{l}\text { Higher } P_{\mathrm{N}}, g_{\mathrm{s}}, \\
C_{\mathrm{i}}, \text { WUE }\end{array}$ & - & $\begin{array}{l}\text { Naz et al. } \\
(2015)\end{array}$ \\
\hline & $\begin{array}{l}\text { Nutrient } \\
\text { solution }\end{array}$ & $\begin{array}{l}0.1-0.3 \mathrm{mg}\left(\mathrm{Na}_{2} \mathrm{SeO}_{3}\right. \\
\left.\text { or } \mathrm{Na}_{2} \mathrm{SeO}_{4}\right) \mathrm{L}^{-1}\end{array}$ & $\begin{array}{l}\text { Helianthus } \\
\text { annuus } \mathrm{L} .\end{array}$ & No stress & $\begin{array}{l}\text { Higher } P_{\mathrm{N}}, E \\
\mathrm{~F}_{\mathrm{v}} / \mathrm{F}_{\mathrm{m}} \text {, and } \mathrm{F}_{\mathrm{v}} / \mathrm{F}_{0}\end{array}$ & - & $\begin{array}{l}\text { Garousi et al. } \\
\text { (2016) }\end{array}$ \\
\hline & Leaf & $\begin{array}{l}10,50 \text { and } 100 \\
\mu \mathrm{g}\left(\mathrm{Na}_{2} \mathrm{SeO}_{4}\right) \mathrm{ml}^{-1}\end{array}$ & $\begin{array}{l}\text { Brassica } \\
\text { oleracea } \mathrm{L} .\end{array}$ & No stress & Higher $F_{m}$ & $\begin{array}{l}0.28-1.41 \mathrm{mg} \\
\mathrm{kg}^{-1}(\text { leaf DM) }\end{array}$ & $\begin{array}{l}\text { Ghasemi et al. } \\
\text { (2016) }\end{array}$ \\
\hline & Substrate & $5 \mathrm{mg}\left(\mathrm{Na}_{2} \mathrm{SeO}_{4}\right) \mathrm{kg}^{-1}$ & $\begin{array}{l}\text { Helianthus } \\
\text { annuus L. }\end{array}$ & Salt & Higher $F_{v} / F_{0}$ & $\begin{array}{l}2.40-2.58 \mathrm{mg} \\
\mathrm{kg}^{-1}(\mathrm{DM}) \text { in } \\
\text { shoots and roots, } \\
\text { respectively }\end{array}$ & $\begin{array}{l}\text { Habibi } \\
(2017 a)\end{array}$ \\
\hline & $\begin{array}{l}\text { Nutrient } \\
\text { solution }\end{array}$ & $1 \mathrm{mg}\left(\mathrm{Na}_{2} \mathrm{SeO}_{4}\right) \mathrm{L}^{-1}$ & $\begin{array}{l}\text { Petroselinum } \\
\text { crispum L. }\end{array}$ & Salt & Higher $\mathrm{F}_{\mathrm{v}} / \mathrm{F}_{\mathrm{m}}$ & - & $\begin{array}{l}\text { Habibi } \\
\text { (2017b) }\end{array}$ \\
\hline & $\begin{array}{l}\text { Nutrient } \\
\text { solution }\end{array}$ & $1 \mu \mathrm{M} \mathrm{Na}_{2} \mathrm{SeO}_{3}$ & Zea mays L. & Salt & $\begin{array}{l}\text { Higher } P_{\mathrm{N}}, g_{\mathrm{s}} \text {, } \\
\text { and } E\end{array}$ & - & $\begin{array}{l}\text { Jiang et al. } \\
(2017)\end{array}$ \\
\hline & $\begin{array}{l}\text { Nutrient } \\
\text { solution }\end{array}$ & $1.5,6 \mu \mathrm{M} \mathrm{Na}_{2} \mathrm{SeO}_{3}$ & $\begin{array}{l}\text { Vicia } \\
\text { faba } \mathrm{L} \text {. }\end{array}$ & Lead & Higher Chl & $\begin{array}{l}4.69-4.67 \mathrm{mg} \\
\mathrm{kg}^{-1}(\text { shoot DM) }\end{array}$ & $\begin{array}{l}\text { Mroczek- } \\
\text { Zdyrska et al. }\end{array}$ \\
\hline
\end{tabular}




\begin{tabular}{|c|c|c|c|c|c|c|}
\hline Substrate & $\begin{array}{l}0.5,1 \text {, and } 2 \mathrm{mg}(\mathrm{Se}) \\
\mathrm{kg}^{-1}\end{array}$ & $\begin{array}{l}\text { Oryza } \\
\text { sativa } \mathrm{L} .\end{array}$ & Drought & $\begin{array}{l}\text { Higher } P_{\mathrm{N}} \\
\text { and } E\end{array}$ & $\begin{array}{l}0.5-2.25 \mathrm{mg} \mathrm{pot}^{-1} \\
\text { in aerial part }\end{array}$ & $\begin{array}{l}\text { Andrade et al. } \\
\text { (2018) }\end{array}$ \\
\hline Leaf & $40 \mathrm{mg}\left(\mathrm{Na}_{2} \mathrm{SeO}_{3}\right) \mathrm{L}^{-1}$ & $\begin{array}{l}\text { Oryza } \\
\text { sativa } \mathrm{L} .\end{array}$ & No stress & $\begin{array}{l}\text { Higher } g_{\mathrm{s}} \\
\text { and } E\end{array}$ & - & $\begin{array}{l}\text { Gao et al. } \\
\text { (2018) }\end{array}$ \\
\hline Leaf & $\begin{array}{l}20,40 \mathrm{mg}\left(\mathrm{Na}_{2} \mathrm{SeO}_{4}\right) \\
\mathrm{L}^{-1}\end{array}$ & Zea mays $\mathrm{L}$. & Salt & Higher Chl & - & $\begin{array}{l}\text { Ashraf et al. } \\
\text { (2018) }\end{array}$ \\
\hline $\begin{array}{l}\text { Nutrient } \\
\text { solution }\end{array}$ & $\begin{array}{l}10 \mu \mathrm{M} \\
\mathrm{Na}_{2} \mathrm{SeO}_{3} \cdot 5 \mathrm{H}_{2} \mathrm{O}\end{array}$ & $\begin{array}{l}\text { Solanum } \\
\text { lycopersicum L. }\end{array}$ & Cadmium & $\begin{array}{l}\text { Higher Chl, } \\
\text { Car, } P_{\mathrm{N}}, g_{\mathrm{s}}, E, \\
\mathrm{~F}_{\mathrm{v}} / \mathrm{F}_{\mathrm{m}}, \Phi_{\mathrm{PSII}}, \\
\text { and } \mathrm{q}_{\mathrm{P}}\end{array}$ & $\begin{array}{l}0.15-0.49 \mu \mathrm{mol} \\
\mathrm{g}^{-1}(\text { shoot DM), } \\
0.14-0.30 \mu \mathrm{mol} \\
\mathrm{g}^{-1}(\text { root DM }) \text {, and } \\
1.12-2.41 \mu \mathrm{mol} \\
\mathrm{g}^{-1}(\text { leaf DM })\end{array}$ & $\begin{array}{l}\text { Alyemeni et al. } \\
\text { (2018) }\end{array}$ \\
\hline $\begin{array}{l}\text { Nutrient } \\
\text { solution }\end{array}$ & $5 \mathrm{ppm} \mathrm{Na}_{2} \mathrm{SeO}_{4}$ & $\begin{array}{l}\text { Allium } \\
\text { sativum } \mathrm{L} .\end{array}$ & Cadmium & $\begin{array}{l}\text { Higher } P_{\mathrm{N}}, E \text {, } \\
\text { and } g_{\mathrm{s}}\end{array}$ & - & $\begin{array}{l}\text { Sepehri and } \\
\text { Gharehbaghli } \\
\text { (2019) }\end{array}$ \\
\hline $\begin{array}{l}\text { Nutrient } \\
\text { solution }\end{array}$ & $4,6,8 \mathrm{mg}\left(\mathrm{SeCl}_{2}\right) \mathrm{L}^{-1}$ & $\begin{array}{l}\text { Caspicum } \\
\text { annum } \mathrm{L} .\end{array}$ & Heat & $\begin{array}{l}\text { Higher } \mathrm{F}_{\mathrm{v}} / \mathrm{F}_{\mathrm{m}} \text {, } \\
E, g_{\mathrm{s}} \text {, and } C_{\mathrm{i}}\end{array}$ & $\begin{array}{l}1.78-3.51 \mathrm{mg} \\
\mathrm{kg}^{-1}(\text { leaf DM), } \\
0.98-1.18 \mathrm{mg} \\
\mathrm{kg}^{-1}(\text { root DM), } \\
0.15-1.85 \mathrm{mg} \\
\mathrm{kg}^{-1} \text { (fruit DM) }\end{array}$ & $\begin{array}{l}\text { Haghighi et al. } \\
\text { (2019) }\end{array}$ \\
\hline Leaf & $\begin{array}{l}10,20,30,40, \text { and } \\
50 \mu \mathrm{M} \mathrm{Na}_{2} \mathrm{SeO}_{4}\end{array}$ & $\begin{array}{l}\text { Oryza } \\
\text { sativa L. }\end{array}$ & No stress & $\begin{array}{l}\text { Higher } P_{\mathrm{N}} \\
\text { and } g_{\mathrm{s}}\end{array}$ & $\begin{array}{l}20-50 \text { ng } \\
\mathrm{g}^{-1}(\text { grain DM }) \\
20-70 \mathrm{ng} \\
\mathrm{g}^{-1}(\text { leaf DM }) \\
12 \mathrm{ng} \mathrm{g}^{-1}(\text { stem DM })\end{array}$ & $\begin{array}{l}\text { Luo et al. } \\
\text { (2019) }\end{array}$ \\
\hline Substrate & $\begin{array}{l}2,4,6 \mu \mathrm{M}\left(\mathrm{Na}_{2} \mathrm{SeO}_{4}\right) \\
\mathrm{kg}^{-1}\end{array}$ & $\begin{array}{l}\text { Brassica } \\
\text { juncea } \mathrm{L} .\end{array}$ & Chromium & $\begin{array}{l}\text { Higher Chl, Car, } \\
P_{\mathrm{N}}, g_{\mathrm{s}}, C_{\mathrm{i}}, E\end{array}$ & & $\begin{array}{l}\text { Handa et al. } \\
\text { (2019) }\end{array}$ \\
\hline $\begin{array}{l}\text { Nutrient } \\
\text { solution }\end{array}$ & $\begin{array}{l}2,4,8 \\
\mathrm{mg}\left(\mathrm{Na}_{2} \mathrm{SeO}_{3} \cdot 5 \mathrm{H}_{2} \mathrm{O}\right) \\
\mathrm{L}^{-1}\end{array}$ & $\begin{array}{l}\text { Raphanus } \\
\text { sativus } \mathrm{L} \text {. }\end{array}$ & Cadmium & $\begin{array}{l}\text { Higher } \Phi_{\text {PSII }} \\
\text { and } F_{v} / F_{m}\end{array}$ & $\begin{array}{l}49.3-124.5 \mathrm{mg} \\
\mathrm{g}^{-1}(\text { root DM }), \\
127.83-443.93 \mathrm{mg} \\
\mathrm{g}^{-1}(\text { leaf DM })\end{array}$ & $\begin{array}{l}\text { Amirabad et al. } \\
(2020)\end{array}$ \\
\hline $\begin{array}{l}\text { Nutrient } \\
\text { solution }\end{array}$ & $\begin{array}{l}1 \mu \mathrm{M} \mathrm{Na}_{2} \mathrm{SeO}_{3} \text { or } \\
\mathrm{Na}_{2} \mathrm{SeO}_{4}\end{array}$ & $\begin{array}{l}\text { Solanum } \\
\text { lycopersicum L. }\end{array}$ & Cadmium & $\begin{array}{l}\text { Higher } E, g_{\mathrm{s}} \text {, } \\
\text { and } P_{\mathrm{N}}\end{array}$ & - & $\begin{array}{l}\text { Alves et al. } \\
(2020)\end{array}$ \\
\hline
\end{tabular}

et al. 2015, Hussain et al. 2021). Table 2 shows that one of the most frequent consequence induced by foliar and substrate treatments with $\mathrm{Si}$ is the improvement of $g_{\mathrm{s}}$ with the parallel enhancement of $P_{\mathrm{N}}$ in both optimal and stressful conditions.

Hussain et al. (2021) found that Si, even in low amounts, increased $P_{\mathrm{N}}, g_{\mathrm{s}}$, and $E$ in Glycine max subjected to low light conditions, reducing $C_{\mathrm{i}}$ and increasing chlorophyll (Chl) content. These effects of $\mathrm{Si}$ are reported in plants grown in shade conditions and the authors related the enhanced photosynthetic rate to the reduction in the leaf area, the increase in fresh mass (FM) and leaf thickness. Similar ameliorative effects on photosynthesis were observed by Tripathi et al. (2021) in G. max. However, in this case, the increase in photosynthetic rate was related to an improved root system suggesting that $\mathrm{Si}$ enhanced water and mineral uptake from the soil. Furthermore, in Si hyperaccumulators, such as Oryza sativa, some authors found that Si had a positive effect on photosynthesis by enhancing the $g_{\mathrm{m}}$ (Detmann et al. 2012, Pereira et al. 2020). The $g_{\mathrm{m}}$ reflects the entire diffusive $\mathrm{CO}_{2}$ pathway in the leaf, from the substomatal air spaces to the carboxylation sites localised in the chloroplast stroma. Obviously, from stomata to chloroplast, $\mathrm{CO}_{2}$ crosses different biophysical barriers such as intercellular air spaces and cell wall
(Gago et al. 2020). Although there is evidence that Si might modify the cell wall thickening (Yamamoto et al. 2012), how Si contributes to both anatomical and biochemical components of $g_{\mathrm{m}}$ remains far to be clear. Future studies related to this aspect might help understand $\mathrm{Si}$-photosynthesis interactions.

\section{Sodium}

As it emerges from the literature, $\mathrm{Na}$ can be beneficial or even essential mainly for $\mathrm{C}_{4} / \mathrm{CAM}$, halophyte, and natrophilic species (Pilon-Smits et al. 2009, Broadley et al. 2012, Maathuis 2014, Kaur et al. 2016). Martínez et al. (2005) reported higher values in $P_{\mathrm{N}}$ and increase in WUE levels in Na-treated plants (with respect to controls) of Atriplex halimus $\left(\mathrm{C}_{4}\right.$ xerohalophyte species $)$ under water stress (Table 3). Moreover, in Panicum antidotale, Hussain et al. (2020) observed an alleviation of the negative impact of drought on gas-exchange parameters in $\mathrm{Na}$ plants $(100 \mathrm{mM} \mathrm{NaCl})$ by maintaining higher values in $P_{\mathrm{N}}$, $g_{\mathrm{s}}, E, V_{\text {cmax }}$, and $J_{\max }$ with respect to drought Na-untreated plants. These beneficial effects are related to a positive impact of the low $\mathrm{Na}$ concentration on photochemical reactions and, in particular, stimulating photosynthetic pigments and photochemical PSII efficiency. This could 
Table 2. Effect of silicon ( $\mathrm{Si})$ on plant photosynthetic traits.

\begin{tabular}{|c|c|c|c|c|c|c|c|}
\hline $\begin{array}{l}\text { Beneficial } \\
\text { element }\end{array}$ & $\begin{array}{l}\text { Administra- } \\
\text { tion method }\end{array}$ & $\begin{array}{l}\text { Chemical form and } \\
\text { concentration }\end{array}$ & Species & Stress type & Observation & $\begin{array}{l}\text { Concentration } \\
\text { in plant tissues }\end{array}$ & Reference \\
\hline \multirow[t]{9}{*}{ Silicon } & $\begin{array}{l}\text { Nutrient } \\
\text { solution }\end{array}$ & $2 \mathrm{mM} \mathrm{Si}$ & \multirow{2}{*}{$\begin{array}{l}\text { Oryza } \\
\text { sativa } \mathrm{L} . \\
\text { Oryza } \\
\text { sativa } \mathrm{L} .\end{array}$} & No stress & $\begin{array}{l}\text { Higher } P_{\mathrm{N}} \\
\text { and } g_{\mathrm{m}}\end{array}$ & $\begin{array}{l}47-51 \mathrm{~g} \\
\mathrm{~kg}^{-1}(\text { leaf DM) }\end{array}$ & $\begin{array}{l}\text { Detmann et al. } \\
\text { (2012) }\end{array}$ \\
\hline & $\begin{array}{l}\text { Nutrient } \\
\text { solution }\end{array}$ & $\begin{array}{l}1.5 \mathrm{mM} \text { added as } \\
\mathrm{K}_{2} \mathrm{SiO}_{3}\end{array}$ & & Zinc & $\begin{array}{l}\text { Increase of } \\
\text { expression of } \\
\text { genes associated } \\
\text { with photosyn- } \\
\text { thesis }\end{array}$ & - & $\begin{array}{l}\text { Song et al. } \\
\text { (2014) }\end{array}$ \\
\hline & Leaf & $6 \mathrm{mM} \mathrm{Na}_{2} \mathrm{SiO}_{7}$ & $\begin{array}{l}\text { Triticum } \\
\text { aestivum } \mathrm{L} .\end{array}$ & Drought & $\begin{array}{l}\text { Higher } P_{\mathrm{N}} \\
\text { and } g_{\mathrm{s}}\end{array}$ & - & $\begin{array}{l}\text { Maghsoudi et al. } \\
\text { (2016) }\end{array}$ \\
\hline & Substrate & $\begin{array}{l}100-500 \mathrm{mg}\left(\mathrm{CaO} \cdot \mathrm{SiO}_{2}\right) \\
\mathrm{L}^{-1}\end{array}$ & \multirow{2}{*}{$\begin{array}{l}\text { Saccharum } \\
\text { officinarum L. } \\
\text { Sorghum } \\
\text { bicolor (L.) } \\
\text { Moench }\end{array}$} & Drought & $\begin{array}{l}\text { Higher } P_{\mathrm{N}} \\
\text { and } g_{\mathrm{s}}\end{array}$ & $\begin{array}{l}1-4 \mathrm{mg} \\
\mathrm{g}^{-1}(\text { leaf DM })\end{array}$ & $\begin{array}{l}\text { Verma et al. } \\
(2020)\end{array}$ \\
\hline & Substrate & $2 \mathrm{mM} \mathrm{Si}$ added as $\mathrm{SiO}_{2}$ & & Drought & $\begin{array}{l}\text { Higher } P_{\mathrm{N}}, g_{\mathrm{s}} \text {, } \\
\text { and } P_{\mathrm{N}} / C_{\mathrm{i}}\end{array}$ & $\begin{array}{l}382-417 \mathrm{mg} \\
\mathrm{kg}^{-1}(\text { leaf DM })\end{array}$ & $\begin{array}{l}\text { Avila et al. } \\
(2020)\end{array}$ \\
\hline & $\begin{array}{l}\text { Nutrient } \\
\text { solution }\end{array}$ & $2 \mathrm{mM} \mathrm{Si}$ & $\begin{array}{l}\text { Oryza } \\
\text { sativa } \mathrm{L} .\end{array}$ & Biotic & $\begin{array}{l}\text { Higher } P_{\mathrm{N}} \\
\text { and } g_{\mathrm{m}}\end{array}$ & $\begin{array}{l}18-20 \mathrm{~g} \\
\mathrm{~kg}^{-1}(\text { leaf DM })\end{array}$ & $\begin{array}{l}\text { Pereira et al. } \\
(2020)\end{array}$ \\
\hline & Substrate & $1 \mathrm{~g}\left(\mathrm{CaO} \cdot \mathrm{SiO}_{2}\right) \mathrm{kg}^{-1}$ (soil) & $\begin{array}{l}\text { Piper } \\
\text { nigrum L. }\end{array}$ & Biotic & $\begin{array}{l}\text { Higher } P_{\mathrm{N}}, g_{\mathrm{s}} \text {, } \\
\text { and WUE }\end{array}$ & - & $\begin{array}{l}\text { D'Addazio et al. } \\
(2020)\end{array}$ \\
\hline & $\begin{array}{l}\text { Substrate } \\
\text { and leaf }\end{array}$ & $\begin{array}{l}\text { Substrate: } 0.08 \mathrm{~kg} \mathrm{~m}^{-2} \\
\text { of fertilizer with } 25 \% \\
\text { of } \mathrm{SiO}_{2} \\
\text { Leaf: } 2 \mathrm{mM} \mathrm{Na}_{2} \mathrm{SiO}_{3}\end{array}$ & $\begin{array}{l}\text { Glycine } \\
\max (\mathrm{L} .) \text { Merr. }\end{array}$ & No stress & $\begin{array}{l}\text { Higher } P_{\mathrm{N}} \\
\text { and } g_{\mathrm{s}}\end{array}$ & - & $\begin{array}{l}\text { Tripathi et al. } \\
(2021)\end{array}$ \\
\hline & Leaf & $200 \mathrm{mg}\left(\mathrm{Na}_{2} \mathrm{SiO}_{3}\right) \mathrm{kg}^{-1}$ & $\begin{array}{l}\text { Glycine } \\
\max (\mathrm{L} .) \text { Merr. }\end{array}$ & Low light & $\begin{array}{l}\text { Higher } P_{\mathrm{N}} \\
\text { and } g_{\mathrm{s}}\end{array}$ & $\begin{array}{l}0.17 \mathrm{mg} \\
\mathrm{g}^{-1}(\text { stem FM })\end{array}$ & $\begin{array}{l}\text { Hussain et al. } \\
\text { (2021) }\end{array}$ \\
\hline
\end{tabular}

Table 3. Effect of sodium (Na) on plant photosynthetic traits.

\begin{tabular}{|c|c|c|c|c|c|c|c|}
\hline $\begin{array}{l}\text { Beneficial } \\
\text { element }\end{array}$ & $\begin{array}{l}\text { Administra- } \\
\text { tion method }\end{array}$ & $\begin{array}{l}\text { Chemical form and } \\
\text { concentration }\end{array}$ & Species & Stress type & Observation & $\begin{array}{l}\text { Concentration } \\
\text { in tissues }\end{array}$ & Reference \\
\hline \multirow[t]{5}{*}{ Sodium } & $\begin{array}{l}\text { Nutrient } \\
\text { solution }\end{array}$ & $50 \mathrm{mM} \mathrm{NaCl}$ & $\begin{array}{l}\text { Atriplex } \\
\text { halimus L. }\end{array}$ & Drought & Higher $P_{\mathrm{N}}$ & $\begin{array}{l}400-800 \mu \mathrm{mol} \\
\mathrm{g}^{-1}(\text { leaf DM })\end{array}$ & $\begin{array}{l}\text { Martínez et al. } \\
\text { (2005) }\end{array}$ \\
\hline & $\begin{array}{l}\text { Nutrient } \\
\text { solution }\end{array}$ & $510 \mathrm{mM} \mathrm{NaCl}$ & $\begin{array}{l}\text { Arthrocnemum } \\
\text { macrostachyum } \\
\text { (Moric) C. Koch }\end{array}$ & No stress & $\begin{array}{l}\text { Higher } P_{\mathrm{N}}, g_{\mathrm{s}} \\
\text { and midday } \mathrm{F}_{\mathrm{v}} / \mathrm{F}_{\mathrm{m}}\end{array}$ & $\begin{array}{l}0.1-9 \mathrm{mmol} \\
\mathrm{g}^{-1}(\text { root } \mathrm{DM}) \\
0.5-5 \mathrm{mmol} \\
\mathrm{g}^{-1}(\text { shoot } \mathrm{DM})\end{array}$ & $\begin{array}{l}\text { Redondo-Gómez } \\
\text { et al. (2010) }\end{array}$ \\
\hline & Leaf & $50 \mathrm{mM} \mathrm{NaCl}$ & $\begin{array}{l}\text { Zygophyllum } \\
\text { xanthoxylum } \\
\text { (Bunge) Engl. }\end{array}$ & Drought & $\begin{array}{l}\text { Higher Chl, } P_{\mathrm{N}}, \\
g_{\mathrm{s}}, \mathrm{WUE}, \text { and } \Phi_{\mathrm{PSII}}\end{array}$ & $\begin{array}{l}2.13 \mathrm{mmol} \\
\mathrm{g}^{-1}(\text { leaf } \mathrm{DM})\end{array}$ & $\begin{array}{l}\text { Ma et al. } \\
\text { (2012) }\end{array}$ \\
\hline & $\begin{array}{l}\text { Nutrient } \\
\text { solution }\end{array}$ & $100 \mathrm{mM} \mathrm{NaCl}$ & $\begin{array}{l}\text { Panicum } \\
\text { antidotale Retz. }\end{array}$ & Drought & $\begin{array}{l}\text { Higher } P_{\mathrm{N}}, g_{\mathrm{s}}, C_{\mathrm{i}}, \\
E, V_{\mathrm{cmax}}, \text { and } J_{\max }\end{array}$ & - & $\begin{array}{l}\text { Hussain et al. } \\
(2020)\end{array}$ \\
\hline & $\begin{array}{l}\text { Nutrient } \\
\text { solution }\end{array}$ & $100-200 \mathrm{mM} \mathrm{NaCl}$ & $\begin{array}{l}\text { Beta vulgaris } \\
\text { subsp. maritima L. }\end{array}$ & No stress & Higher $P_{\mathrm{N}}$ & $\begin{array}{l}\text { 190-400 mM } \\
\text { in leaves }\end{array}$ & $\begin{array}{l}\text { Rasouli et al. } \\
\text { (2021) }\end{array}$ \\
\hline
\end{tabular}

be explained by the fact that, in $\mathrm{C}_{4}$ plants, $\mathrm{Na}$ acts as an essential element for the conversion of pyruvate to phosphoenolpyruvate in mesophyll cells, and it is also involved in the transport of pyruvate into mesophyll cell chloroplasts by sodium-dependent pyruvate transporters (Furumoto et al. 2011). Indeed, Furumoto et al. (2011) proposed that sodium-dependent pyruvate transport in some $\mathrm{C}_{4}$ species is regulated by a two-translocator system as follows. Pyruvate enters the chloroplast with $\mathrm{Na}$ by a sodium/pyruvate cotransporter (BASS2). On the other hand, a sodium $/ \mathrm{H}^{+}$antiporter (NHD1) exchanges protons (required for the conversion of phosphoenolpyruvate) with sodium cations. Pyruvate is an essential molecule for many plastid-localized metabolic pathways of plants, including the $\mathrm{C}_{4}$ photosynthetic $\mathrm{CO}_{2}$-concentrating mechanism (Ohnishi et al. 1990, Furumoto et al. 2011). Sodium, maintaining the granal stacking, also provides a suitable environment for the energy transfer between PSII and PSI (Brownell and Bielig 1996); however, further studies need to be conducted to elucidate the effects of low salinity on photochemical reactions.

Similar ameliorative effects of $\mathrm{Na}$ supplementation 
under drought stress were also observed in Zygophyllum xanthoxylum, a succulent xerophyte woody $\mathrm{C}_{3}$ species (Ma et al. 2012). The authors suggested that this species showed similar properties to salt-accumulating halophytes as the increase in $P_{\mathrm{N}}$ values was strongly related to $g_{\mathrm{s}}$, probably enhanced through Na-mediated osmotic adjustments (Redondo-Gómez et al. 2010, Hedrich and Shabala 2018).

In conclusion, $\mathrm{Na}$ is not only beneficial but essential for some $\mathrm{C}_{4}$ or CAM species but it is beneficial even for some halophytic species that accumulate this element in the salt-resistance mechanism and in natrophilic species or in drought conditions.

\section{Iodine}

Although I biofortification in plants was deeply investigated (Cheng 1981, Blasco et al. 2011, Kiferle et al. 2021, Izydorczyk et al. 2021), there is a lack of information about the ameliorative effects on photosynthesis related to I if compared to other elements used in plant biofortification such as Se (Table 4). This was probably due to the high I toxicity for plants and to the difficulty to find the concentration at which this element can be useful for plant growth. Once inside the plant, I is transported predominantly through the xylem; however, a transport through phloem was also reported suggesting that I is relatively mobile to sink organs (Herrett et al. 1962, Landini et al. 2011, Smoleń et al. 2014).

In an experiment conducted by Blasco et al. (2011), by using low $(20$ and $40 \mu \mathrm{M})$ and high doses $(80 \mu \mathrm{M})$ of I as KI $\left(\mathrm{I}^{-}\right)$or $\mathrm{KIO}_{3}\left(\mathrm{IO}_{3}^{-}\right)$, it was observed that the administration of very low doses of $\mathrm{I}^{-}$or $\mathrm{IO}_{3}^{-}$promoted $g_{\mathrm{s}}$ and $E$, that, in turn, enhanced $P_{\mathrm{N}}$ in lettuce plants. However, only plants treated with $\mathrm{IO}_{3}^{-}$(at $80 \mu \mathrm{M}$ ) maintained an elevated $P_{\mathrm{N}}$ in respect to controls, whereas $\mathrm{I}^{-}$induced a decrease in gas-exchange parameters, highlighting the phytotoxic effect of high doses of this I form (Blasco et al. 2011).

To date, a detailed report of Kiferle et al. (2021) using Arabidopsis plants, revealed the existence of several iodinated proteins involved in photosynthesis, such as constituents of PSII (PsbA, PsbB, PsbC, and PsbD), OEC (PsbO, PsbP, PsbQ, and PsbR), LHCII (CAB3, LHCB2.1, LHCB1B1, LHCB3, LHCB5), PSI (PsaB, PsaE, PsaF, and $\mathrm{PsaH})$, Cyt $b_{6} / f$ (PetA and PetC), plastocyanin electron carrier (PETE2), and ferredoxin-NADP $(+)$ oxidoreductase (FNR1). Moreover, other iodinated proteins were involved in the development/maintenance of both photosystems (Psb27, Psb29, Psb31, and Psb33), in PSII photoprotection (MPH1) or in the degradation of the photodamaged D1 reaction centre. Since I can be a structural component of several different proteins involved in photosynthesis, the authors proposed that I could have a potential nutritional role in plants. However, the capability to generate iodinated proteins represents one of the main reasons of the high toxicity exerted by this element and explain why the range of concentrations between positive and negative effects in plants is extremely narrow.

\section{Titanium}

$\mathrm{Ti}$, after $\mathrm{Fe}$, is the most abundant transition metal, and its abundance on Earth's crust is about five times lower than that of Fe and 100 times greater than that of $\mathrm{Cu}$. Ti, usually administrated on leaves as ionic $\mathrm{Ti}$ or $\mathrm{TiO}_{2}$ nanoparticles (NPs), was successfully used as beneficial element to improve crop production (Ercoli et al. 2008, Lyu et al. 2017).

In plants, there is a lack of knowledge about the transport of $\mathrm{Ti}$ and $\mathrm{TiO}_{2} \mathrm{NP}_{\mathrm{S}}$; it is conceivable that they can be absorbed by roots or leaf stomata and actively/ passively transported through plant tissues following a similar transport of Fe (Lyu et al. 2017).

About the beneficial effects of this element in the photosynthetic process, it was observed that Ti application stimulated the Chl content in Vigna radiata (Raliya et al. 2015), Fragaria $\times$ ananassa (Choi et al. 2015), Cucumis sativus (Servin et al. 2013), G. $\max$ (Hussain et al. 2019a), and Dracocephalum moldavica (Gohari et al. 2020) (Table 5). However, the way in which Ti induces an accumulation in $\mathrm{Chl}$ is still an open question. Nonetheless, Ze et al. (2011) observed an increase in PSII LHCII-b gene expression resulting in an enhancement of the energy transport between $\mathrm{Chl} b$ and carotenoid and in this way promoting the distribution of light energy from PSI to PSII by increasing LHCII in Arabidopsis plants treated with a solution of $0.25 \%$ Ti NPs.

It was observed that $\mathrm{Ti}$ and $\mathrm{TiO}_{2} \mathrm{NP}_{\mathrm{S}}$ promoted $P_{\mathrm{N}}$ and $g_{\mathrm{s}}$ under nonlimiting or stressful conditions (Gao et al.

Table 4. Effect of iodine (I) on plant photosynthetic traits.

\begin{tabular}{|c|c|c|c|c|c|c|c|}
\hline $\begin{array}{l}\text { Beneficial } \\
\text { element }\end{array}$ & $\begin{array}{l}\text { Administra- } \\
\text { tion method }\end{array}$ & $\begin{array}{l}\text { Chemical form } \\
\text { and concentration }\end{array}$ & Species & Stress type & Observation & $\begin{array}{l}\text { Concentration } \\
\text { in plant tissues }\end{array}$ & Reference \\
\hline \multirow[t]{2}{*}{ Iodine } & $\begin{array}{l}\text { Nutrient } \\
\text { solution }\end{array}$ & $\begin{array}{l}20,40 \mu \mathrm{mol}(\mathrm{KI}) \mathrm{L}^{-1}, \text { and } \\
20,40,80 \mu \mathrm{mol}\left(\mathrm{KIO}_{3}\right) \mathrm{L}^{-1}\end{array}$ & $\begin{array}{l}\text { Lactuca } \\
\text { sativa } \mathrm{L} \text {. }\end{array}$ & No stress & Higher $P_{\mathrm{N}}, g_{\mathrm{s}}, E$ & - & $\begin{array}{l}\text { Blasco et al. } \\
\text { (2011) }\end{array}$ \\
\hline & $\begin{array}{l}\text { Nutrient } \\
\text { solution }\end{array}$ & 0.20 or $10 \mu \mathrm{M} \mathrm{KIO}_{3}$ & $\begin{array}{l}\text { Arabidopsis } \\
\text { thaliana (L.) } \\
\text { Heynh. }\end{array}$ & No stress & $\begin{array}{l}\text { Presence of } 31 \\
\text { iodinated proteins } \\
\text { involved in } \\
\text { photosynthesis } \\
\text { (constituents of PSII, } \\
\text { PSI, OEC, LHCII, } \\
\text { Cyt } b_{6} / f \text { ) }\end{array}$ & - & $\begin{array}{l}\text { Kiferle et al. } \\
(2021)\end{array}$ \\
\hline
\end{tabular}


Table 5. Effect of titanium (Ti) on plant photosynthetic traits.

\begin{tabular}{|c|c|c|c|c|c|c|c|}
\hline $\begin{array}{l}\text { Beneficial } \\
\text { element }\end{array}$ & $\begin{array}{l}\text { Administra- } \\
\text { tion method }\end{array}$ & $\begin{array}{l}\text { Chemical form and } \\
\text { concentration }\end{array}$ & Species & Stress type & Observation & $\begin{array}{l}\text { Concentration } \\
\text { in plant tissues }\end{array}$ & Reference \\
\hline \multirow[t]{9}{*}{ Titanium } & $\begin{array}{l}\text { Nutrient } \\
\text { solution } \\
\text { Leaf }\end{array}$ & $\begin{array}{l}\text { Solution with } 0.03 \% \\
\mathrm{TiO}_{2} \text { NPs }\end{array}$ & $\begin{array}{l}\text { Spinacia } \\
\text { oleracea } \mathrm{L} .\end{array}$ & No stress & $\begin{array}{l}\text { Higher } P_{\mathrm{N}}, \\
\text { higher Rubisco } \\
\text { carboxylase and } \\
\text { activase activity }\end{array}$ & - & $\begin{array}{l}\text { Gao et al. } \\
(2008)\end{array}$ \\
\hline & Leaf & $\begin{array}{l}\text { Solution with } 0.25 \% \\
\mathrm{TiO}_{2} \text { NPs }\end{array}$ & $\begin{array}{l}\text { Arabidopsis } \\
\text { thaliana (L.) } \\
\text { Heynh. }\end{array}$ & No stress & $\begin{array}{l}\text { Increase of LHCII } b \\
\text { gene expression } \\
\text { and LHCII content }\end{array}$ & - & $\begin{array}{l}\text { Ze et al. } \\
\text { (2011) }\end{array}$ \\
\hline & Leaf & 720 ppm $\mathrm{TiO}_{2}$ NPs & $\begin{array}{l}\text { Cucumis } \\
\text { sativus } \mathrm{L} \text {. }\end{array}$ & No stress & $\begin{array}{l}\text { Increase in Chl } \\
\text { index (SPAD) }\end{array}$ & - & $\begin{array}{l}\text { Servin et al. } \\
(2013)\end{array}$ \\
\hline & Leaf & $50-100 \mathrm{mg}\left(\mathrm{TiO}_{2}\right) \mathrm{kg}^{-1}$ & $\begin{array}{l}\text { Fragaria } \times \\
\text { ananassa } \text { Duch. }\end{array}$ & No stress & $\begin{array}{l}\text { Increase in } \mathrm{Chl} \\
\text { content }\end{array}$ & - & $\begin{array}{l}\text { Choi et al. } \\
\text { (2015) }\end{array}$ \\
\hline & Leaf & $10 \mathrm{mg}\left(\mathrm{TiO}_{2} \mathrm{NPs}\right) \mathrm{L}^{-1}$ & $\begin{array}{l}\text { Vigna } \\
\text { radiata L. }\end{array}$ & No stress & $\begin{array}{l}\text { Increase in } \mathrm{Chl} \\
\text { content }\end{array}$ & - & $\begin{array}{l}\text { Raliya et al. } \\
\text { (2015) }\end{array}$ \\
\hline & Leaf & $\begin{array}{l}12.5-25 \mathrm{mg}(\text { ionic } \mathrm{Ti}) \\
\mathrm{L}^{-1}\end{array}$ & $\begin{array}{l}\text { Glycine } \\
\max (\mathrm{L} .) \text { Merr. }\end{array}$ & Low light & $\begin{array}{l}\text { Higher Chl, } \Phi_{\mathrm{PSII}}, \\
\mathrm{q}_{\mathrm{p}}, P_{\mathrm{N}}, g_{\mathrm{s}} \text {, and } E\end{array}$ & $\begin{array}{l}25-40 \mathrm{mg} \\
\mathrm{kg}^{-1}(\text { leaf DM), } \\
12.5-35 \mathrm{mg} \\
\mathrm{kg}^{-1}(\text { stem DM), } \\
12.5-150 \mathrm{mg} \\
\mathrm{kg}^{-1} \text { (root DM) }\end{array}$ & $\begin{array}{l}\text { Hussain et al. } \\
\text { (2019a) }\end{array}$ \\
\hline & Leaf & $\begin{array}{l}2.5 \mathrm{mg} \text { (ionic Ti) } \\
\text { plant }^{-1}\end{array}$ & $\begin{array}{l}\text { Glycine } \\
\max (\mathrm{L} .) \text { Merr. }\end{array}$ & Low light & $\begin{array}{l}\text { Higher ETR, } \\
\mathrm{F}_{\mathrm{v}} / \mathrm{F}_{\mathrm{m}}, \mathrm{Chl}, \\
\text { Rubisco activase } \\
\text { activity }\end{array}$ & $\begin{array}{l}35-100 \mathrm{mg} \\
\mathrm{kg}^{-1}(\text { root DM }) \\
2-12 \mathrm{mg} \\
\mathrm{kg}^{-1}(\text { stem DM }), \\
\text { and } 20-80 \mathrm{mg} \\
\mathrm{kg}^{-1}(\text { leaf DM })\end{array}$ & $\begin{array}{l}\text { Hussain et al. } \\
(2019 b)\end{array}$ \\
\hline & $\begin{array}{l}\text { Nutrient } \\
\text { solution }\end{array}$ & $\begin{array}{l}50-100 \mathrm{mg}\left(\mathrm{TiO}_{2} \mathrm{NPs}\right) \\
\mathrm{L}^{-1}\end{array}$ & $\begin{array}{l}\text { Dracocephalum } \\
\text { moldavica } \mathrm{L} .\end{array}$ & No stress & $\begin{array}{l}\text { Higher Chl, } \mathrm{F}_{\mathrm{v}} / \mathrm{F}_{\mathrm{m}} \text {, } \\
\text { and } \Phi_{\mathrm{PSII}}\end{array}$ & - & $\begin{array}{l}\text { Gohari et al. } \\
\text { (2020) }\end{array}$ \\
\hline & Leaf & $\begin{array}{l}10-1500 \mathrm{mg}\left(\mathrm{TiO}_{2}\right. \\
\text { NPs and } \mu \mathrm{Ps}) \mathrm{L}^{-1}\end{array}$ & $\begin{array}{l}\text { Raphanus } \\
\text { sativus L. }\end{array}$ & No stress & $\begin{array}{l}\text { Transient increase } \\
\text { of } P_{\mathrm{N}} \text { and } g_{\mathrm{s}}\end{array}$ & $\begin{array}{l}10-80 \mathrm{mg} \\
\mathrm{kg}^{-1}(\mathrm{DM}) \text { in } \\
\text { leaves treated } \\
\text { with NPs and } \\
10-225 \mathrm{mg} \\
\mathrm{kg}^{-1}(\mathrm{DM}) \text { in } \\
\text { leaves treated } \\
\text { with } \mu \mathrm{Ps}\end{array}$ & $\begin{array}{l}\text { Tighe-Neira } \\
\text { et al. }(2020)\end{array}$ \\
\hline
\end{tabular}

2008, Hussain et al. 2019a, Tighe-Neira et al. 2020). In addition, other positive effects of $\mathrm{Ti}$ or $\mathrm{TiO}_{2} \mathrm{NP}_{\mathrm{S}}$ on photosynthetic traits, such as a higher $\mathrm{F}_{\mathrm{v}} / \mathrm{F}_{\mathrm{m}}, \Phi_{\mathrm{PSII}}, \mathrm{ETR}$, and $\mathrm{q}_{\mathrm{p}}$, have been reported in Dracocephalum moldavica and G. $\max$ (Hussain et al. 2019a,b; Gohari et al. 2020). The increase of photosynthetic rate is likely linked to the capability of Ti-treated plants to increase the rate of Rubisco activase and the carboxylation activity of Rubisco (Gao et al. 2008, Hussain et al. 2019b). The promising beneficial roles exerted by Ti on leaf photosynthesis could open up new research lines in plant nutrition, fertilization, and biofortification.

\section{Cerium and lanthanum}

Cerium and lanthanum belong to the group of rare earth elements and possess unique photo-electromagnetic effects on photosynthesis (Liu et al. 2009). Ce $\mathrm{NP}_{\mathrm{S}}$ can be absorbed by the leaves through stomatal apertures or by roots but are difficult to be translocated within the plant (Birbaum et al. 2010, Zhang et al. 2011). Nonetheless, Ma et al. (2015) demonstrated Ce $\mathrm{NP}_{\mathrm{S}}$ were translocated to aerial parts in the form of $\mathrm{CeO}_{3}(\sim 30 \%)$ or combined with carboxyl group $(\sim 70 \%)$. Once transported into the mesophyll cell, Ce enters the chloroplast via nonendocytic pathways, influenced by the electrochemical gradient of the plasma membrane potential (Wu et al. 2017). Ma et al. (2015) also observed that, in plant roots, about $80 \%$ of $\mathrm{La} \mathrm{NP}$ sere transformed into La carboxylates and phosphates, and only these two forms were found in leaves at similar abundances. In general, the supply of Ce or La to plants, grown with or without stress, improves the $P_{\mathrm{N}}$ values, in some cases due to an increase in $g_{\mathrm{s}}$, as it was reported in La-treated plants of Amorphophallus sinensis (Li et al. 2020), Ce-treated plants of Phaseolus vulgaris and S. bicolor (Djanaguiraman et al. 2018, Salgado et al. 2020), or in La-treated Z. mays plants (Cui et al. 2019) (Table 6). Moreover, Wu et al. (2017) reported that 
Table 6. Effect of lanthanum (La) and cerium (Ce) on plant photosynthetic traits.

\begin{tabular}{|c|c|c|c|c|c|c|c|}
\hline $\begin{array}{l}\text { Beneficial } \\
\text { element }\end{array}$ & $\begin{array}{l}\text { Administra- } \\
\text { tion method }\end{array}$ & $\begin{array}{l}\text { Chemical form } \\
\text { and concentration }\end{array}$ & Species & Stress type & Observation & $\begin{array}{l}\text { Concentrations } \\
\text { in plant tissues }\end{array}$ & Reference \\
\hline \multirow[t]{4}{*}{ Lanthanum } & $\begin{array}{l}\text { Nutrient } \\
\text { solution } \\
\text { Leaf }\end{array}$ & $500 \mu \mathrm{mol}\left(\mathrm{CeCl}_{3}\right) \mathrm{L}^{-1}$ & $\begin{array}{l}\text { Spinacia } \\
\text { oleracea } \mathrm{L} .\end{array}$ & No stress & $\begin{array}{l}\text { Promotes the } \\
\text { energy transfer } \\
\text { from LHCII to } \\
\text { PSII and } \\
\text { accelerates water } \\
\text { photolysis and } \mathrm{O}_{2} \\
\text { evolution }\end{array}$ & - & $\begin{array}{l}\text { Liu et al. } \\
\text { (2007b) }\end{array}$ \\
\hline & Leaf & $100 \mathrm{mg}(\mathrm{La}) \mathrm{L}^{-1}$ & $\begin{array}{l}\text { Pseudostellaria } \\
\text { heterophylla } \\
\text { (Miquel) Pax }\end{array}$ & No stress & $\begin{array}{l}\text { Higher } P_{\mathrm{N}}, g_{\mathrm{s}} \\
\mathrm{F}_{\mathrm{v}} / \mathrm{F}_{\mathrm{m}}, \text { and } \Phi_{\mathrm{PSII}}\end{array}$ & - & $\begin{array}{l}\text { Ma et al. } \\
\text { (2017) }\end{array}$ \\
\hline & $\begin{array}{l}\text { Nutrient } \\
\text { solution }\end{array}$ & $\begin{array}{l}400-800 \mu \mathrm{mol}\left(\mathrm{LaCl}_{3}\right) \\
\mathrm{L}^{-1}\end{array}$ & Zea mays L. & No stress & $\begin{array}{l}\text { Higher Chl, Car, } \\
P_{\mathrm{N}}, g_{\mathrm{s}}, \mathrm{F}_{\mathrm{v}} / \mathrm{F}_{\mathrm{m}} \text {, and } \\
\Phi_{\mathrm{PSII}}\end{array}$ & - & $\begin{array}{l}\text { Cui et al. } \\
(2019)\end{array}$ \\
\hline & Leaf & $160 \mathrm{mg}\left(\mathrm{LaCl}_{3}\right) \mathrm{L}^{-1}$ & $\begin{array}{l}\text { Amorphophallus } \\
\text { sinensis Belval }\end{array}$ & No stress & $\begin{array}{l}\text { Higher Chl, Car, } \\
P_{\mathrm{N}} \text {, and } g_{\mathrm{s}}\end{array}$ & - & $\begin{array}{l}\text { Li et al. } \\
(2020)\end{array}$ \\
\hline \multirow[t]{5}{*}{ Cerium } & $\begin{array}{l}\text { Nutrient } \\
\text { solution } \\
\text { Leaf }\end{array}$ & $20 \mu \mathrm{mol}\left(\mathrm{CeCl}_{3}\right) \mathrm{L}^{-1}$ & $\begin{array}{l}\text { Spinacia } \\
\text { oleracea } \mathrm{L} .\end{array}$ & No stress & $\begin{array}{l}\text { Promotes the } \\
\text { energy transfer } \\
\text { from LHCII to } \\
\text { PSII and } \\
\text { accelerates water } \\
\text { photolysis and } \mathrm{O}_{2} \\
\text { evolution }\end{array}$ & - & $\begin{array}{l}\text { Liu et al. } \\
\text { (2007b) }\end{array}$ \\
\hline & $\begin{array}{l}\text { Nutrient } \\
\text { solution } \\
\text { Leaf }\end{array}$ & $20 \mu \mathrm{mol}\left(\mathrm{CeCl}_{3}\right) \mathrm{L}^{-1}$ & $\begin{array}{l}\text { Arabidopsis } \\
\text { thaliana (L.) } \\
\text { Heynh. }\end{array}$ & No stress & $\begin{array}{l}\text { Higher Chl, } \\
\text { increase of } \\
\text { LHCII } b \text { gene } \\
\text { expression and } \\
\text { LHCII content }\end{array}$ & - & $\begin{array}{l}\text { Liu et al. } \\
\text { (2009) }\end{array}$ \\
\hline & Leaf & $\begin{array}{l}\mathrm{CeO}_{2} \mathrm{NPs} \text { with low } \\
\mathrm{Ce}^{3+} / \mathrm{Ce}^{4+} \text { ratio }\end{array}$ & $\begin{array}{l}\text { Arabidopsis } \\
\text { thaliana (L.) } \\
\text { Heynh. }\end{array}$ & $\begin{array}{l}\text { Heat } \\
\text { High light } \\
\text { Chilling }\end{array}$ & $\begin{array}{l}\text { Higher } P_{\mathrm{N}}, V_{\mathrm{cmax}}, \\
\mathrm{F}_{\mathrm{v}} / \mathrm{F}_{\mathrm{m}} \text {, and } \Phi_{\mathrm{PSII}}\end{array}$ & - & $\begin{array}{l}\text { Wu et al. } \\
\text { (2017) }\end{array}$ \\
\hline & Leaf & $10 \mathrm{mg}\left(\mathrm{CeO}_{2} \mathrm{NPs}\right) \mathrm{L}^{-1}$ & $\begin{array}{l}\text { Sorghum } \\
\text { bicolor (L.) } \\
\text { Moench }\end{array}$ & Drought & $\begin{array}{l}\text { Higher } \mathrm{Chl} \text { index } \\
(\mathrm{SPAD}), P_{\mathrm{N}}, g_{\mathrm{s}} \\
\text { and } \mathrm{F}_{\mathrm{v}} / \mathrm{F}_{\mathrm{m}}\end{array}$ & $\begin{array}{l}5.1-10 \mathrm{mg} \\
\mathrm{kg}^{-1}(\text { root FM }) \\
\text { and } 4-4.5 \mathrm{mg} \\
\mathrm{kg}^{-1}(\text { shoot FM })\end{array}$ & $\begin{array}{l}\text { Djanaguiraman } \\
\text { et al. }(2018)\end{array}$ \\
\hline & $\begin{array}{l}\text { Nutrient } \\
\text { solution }\end{array}$ & $\begin{array}{l}0.1 \mu \mathrm{mol}\left(\mathrm{Ce}\left(\mathrm{NO}_{3}\right)_{3} \cdot 6 \mathrm{H}_{2} \mathrm{O}\right) \\
\mathrm{L}^{-1}\end{array}$ & $\begin{array}{l}\text { Phaseolus } \\
\text { vulgaris L. }\end{array}$ & Drought & $\begin{array}{l}\text { Higher Chl, } P_{\mathrm{N}} \text {, } \\
\text { and } g_{\mathrm{s}}\end{array}$ & - & $\begin{array}{l}\text { Salgado et al. } \\
(2020)\end{array}$ \\
\hline
\end{tabular}

in Arabidopsis plants treated with a low $\mathrm{Ce}^{3+} / \mathrm{Ce}^{4+}$ ratio photosynthetic performance improved under stress conditions (excess light, continuous excess light, heat, and dark chilling). The authors also observed that the lower $\mathrm{Ce}^{3+} / \mathrm{Ce}^{4+}$ ratio was able to scavenge $\mathrm{ROS}$, including hydrogen peroxide, superoxide anion, and hydroxyl radicals. The capability of Ce to reduce oxidative stress was also suggested in plants subjected to drought (Djanaguiraman et al. 2018, Salgado et al. 2020).

Besides their capability to increase the Chl content improving the light-harvesting complex (Liu et al. 2007a, Liu et al. 2009, Li et al. 2020), Ce increases light absorption as well as the energy transfer within LHCII and from tyrosine residues on the oxidizing side of PSII II to Chl $a$ in the PSII reaction centres (Liu et al. 2007b). This is probably explained by the results of Hong et al. (2002) in Spinacia oleracea, where they found that La or $\mathrm{Ce}$ can replace $\mathrm{Mg}$ in the $\mathrm{Chl}$ assembling and promote the formation of PSII more significantly than that of PSI.
Conclusion: More attention was focused on the toxic effects of some elements whereas it is evident that some elements are beneficial in plant photosynthetic process (Fig. 1). As previously reported, the mechanisms through which these beneficial elements exert their promoting effects on leaf photosynthesis are far to be fully elucidated. Moreover, in some works, there are no explanations of the physiological/biochemical mechanisms underlying these ameliorative effects.

The regulatory mechanisms in which those elements are involved need to be clarified in condition of plant stress, too; this, in order to establish their possible ameliorative role under abiotic stresses. Only further investigations focused on the physiological and biochemical mechanisms of action of beneficial elements will make possible to fully exploit those elements to promote plant growth, resilience, and productivity. 


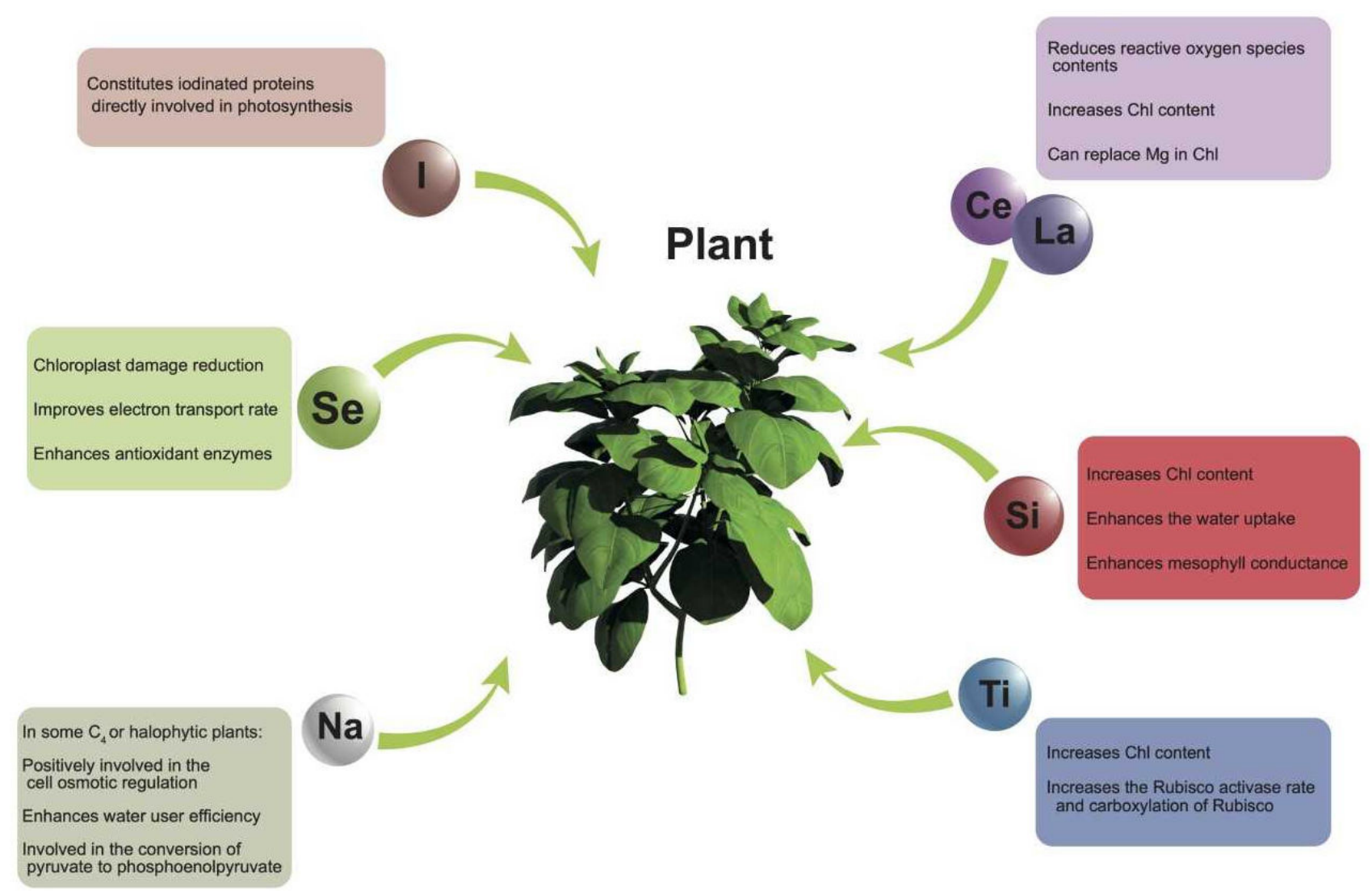

Fig. 1. Potential promoting roles of beneficial elements on plant photosynthetic traits.

\section{References}

Aggarwal A., Sharma I., Tripati B.N. et al.: Metal toxicity and photosynthesis. - In: Ithoh S., Mohanty P., Guruprasad K.N. (ed.): Photosynthesis: Overviews on Recent Progress \& Future Perspectives. Pp. 229-236. IK International Publishing House, New Delhi 2012.

Alves L.R., Rossatto D.R., Rossi M.L. et al.: Selenium improves photosynthesis and induces ultrastructural changes but does not alleviate cadmium-stress damages in tomato plants. Protoplasma 257: 597-605, 2020.

Alyemeni M.N., Ahanger M.A., Wijaya L. et al.: Selenium mitigates cadmium-induced oxidative stress in tomato (Solanum lycopersicum L.) plants by modulating chlorophyll fluorescence, osmolyte accumulation, and antioxidant system. - Protoplasma 255: 459-469, 2018.

Amirabad S.A., Behtash F., Vafaee Y.: Selenium mitigates cadmium toxicity by preventing oxidative stress and enhancing photosynthesis and micronutrient availability on radish (Raphanus sativus L.) cv. Cherry Belle. - Environ. Sci. Pollut. R. 27: 12476-12490, 2020.

Andrade F.R., da Silva G.N., Guimarães K.C. et al.: Selenium protects rice plants from water deficit stress. - Ecotox. Environ. Safe. 164: 562-570, 2018.

Arnon D.I., Stout P.R.: The essentiality of certain elements in minute quantity for plants with special reference to copper. Plant Physiol. 14: 371-375, 1939.

Ashraf M.A., Akbar A., Parveen A. et al:: Phenological application of selenium differentially improves growth, oxidative defense and ion homeostasis in maize under salinity stress. - Plant Physiol. Bioch. 123: 268-280, 2018.

Avila R.G., Magalhães P.C., da Silva E.M. et al.: Silicon supplementation improves tolerance to water deficiency in sorghum plants by increasing root system growth and improving photosynthesis. - Silicon 12: 2545-2554, 2020.

Balakhnina T.I., Nadezhkina E.S.: Effect of selenium on growth and antioxidant capacity of Triticum aestivum L. during development of lead-induced oxidative stress. - Russ. J. Plant Physiol. 64: 215-223, 2017.

Birbaum K., Brogioli R., Schellenberg M. et al.: No evidence for cerium dioxide nanoparticle translocation in maize plants. Environ. Sci. Technol. 44: 8718-8723, 2010.

Blasco B., Rios J.J., Leyva R. et al.: Photosynthesis and metabolism of sugars from lettuce plants (Lactuca sativa $\mathrm{L}$. var. longifolia) subjected to biofortification with iodine. Plant Growth Regul. 65: 137-143, 2011.

Breznik B., Germ M., Gaberscik A., Kreft I.: Combined effects of elevated UV-B radiation and the addition of selenium on common (Fagopyrum esculentum Moench) and tartary [Fagopyrum tataricum (L.) Gaertn.] buckwheat. Photosynthetica 43: 583-589, 2005.

Broadley M., Brown P., Cakmak I. et al.: Beneficial Elements. In: Marschner P. (ed.): Marschner's Mineral Nutrition of Higher Plants. Pp. 249-269. Academic Press, Amsterdam 2012.

Brownell P.F., Bielig L.M.: The role of sodium in the conversion of pyruvate to phosphoenolpyruvate in mesophyll chloroplasts of $\mathrm{C}_{4}$ plants. - Funct. Plant Biol. 23: 171-177, 1996. 
Castillo-Godina R.G., Foroughbakhch-Pournavab R., BenavidesMendoza A.: Effect of selenium on elemental concentration and antioxidant enzymatic activity of tomato plants. - J. Agr. Sci. Tech. 18: 233-244, 2016.

Cheng B.T.: The role of iodine, silicon and titanium on manganese toxicity in an acid soil [in the cultivation of tomatoes and cucumber]. - Agrochimica 25: 268-277, 1981.

Choi H.G., Moon B.Y., Bekhzod K. et al.: Effects of foliar fertilization containing titanium dioxide on growth, yield and quality of strawberries during cultivation. - Hortic. Environ. Biote. 56: 575-581, 2015.

Cui W., Kamran M., Song Q. et al.: Lanthanum chloride improves maize grain yield by promoting photosynthetic characteristics, antioxidants enzymes and endogenous hormone at reproductive stages. - J. Rare Earth. 37: 781-790, 2019.

Detmann K.C., Araújo W.L., Martins S.C.V. et al.: Silicon nutrition increases grain yield, which, in turn, exerts a feedforward stimulation of photosynthetic rates via enhanced mesophyll conductance and alters primary metabolism in rice. - New Phytol. 196: 752-762, 2012.

Djanaguiraman M., Nair R., Giraldo J.P. et al.: Cerium oxide nanoparticles decrease drought-induced oxidative damage in Sorghum leading to higher photosynthesis and grain yield. ACS Omega 3: 14406-14416, 2018.

Djanaguiraman M., Prasad P.V.V., Seppanen M.: Selenium protects sorghum leaves from oxidative damage under high temperature stress by enhancing antioxidant defense system. - Plant Physiol. Bioch. 48: 999-1007, 2010.

D'Addazio V., Silva J.V.G., Jardim A.S. et al.: Silicon improves the photosynthetic performance of black pepper plants inoculated with Fusarium solani f. sp. piperis. - Photosynthetica 58: 692-701, 2020

Epstein E.: Mineral metabolism. - In: Bonner J., Varner J.E. (ed.): Plant Biochemistry. Pp. 438-466. Academic Press, New York 1965.

Ercoli L., Mariotti M., Niccolai L. et al.: The use of titanium shavings as a fertilizer alternative for maize production. Agrochimica 52: 360-376, 2008.

Feng R., Wei C., Tu S.: The roles of selenium in protecting plants against abiotic stresses. - Environ. Exp. Bot. 87: 58-68, 2013.

Feng T., Chen S.S., Gao D.Q. et al.: Selenium improves photosynthesis and protects photosystem II in pear (Pyrus bretschneideri), grape (Vitis vinifera), and peach (Prunus persica). - Photosynthetica 53: 609-612, 2015.

Filek M., Kościelniak J., Łabanowska M. et al.: Selenium-induced protection of photosynthesis activity in rape (Brassica napus) seedlings subjected to cadmium stress. Fluorescence and EPR measurements. - Photosynth. Res. 105: 27-37, 2010.

Furumoto T., Yamaguchi T., Ohshima-Ichie Y. et al.: A plastidial sodium-dependent pyruvate transporter. - Nature 476: 472$475,2011$.

Gago J., Daloso D.M., Carriquí M. et al.: Mesophyll conductance: the leaf corridors for photosynthesis. - Biochem. Soc. T. 48: 429-439, 2020.

Gao F., Liu C., Qu C. et al.: Was improvement of spinach growth by nano- $\mathrm{TiO}_{2}$ treatment related to the changes of Rubisco activase? - BioMetals 21: 211-217, 2008.

Gao M., Zhou J., Liu H. et al.: Foliar spraying with silicon and selenium reduces cadmium uptake and mitigates cadmium toxicity in rice. - Sci. Total Environ. 631-632: 1100-1108, 2018.

Garousi F., Kovács B., Veres S.: Investigation of photosynthesis status of sunflower plants up-taking different forms of selenium. - Adv. Plants Agric. Res. 3: 00083, 2016.

Germ M., Kreft I., Stibilj V., Urbanc-Berčič O.: Combined effects of selenium and drought on photosynthesis and mitochondrial respiration in potato. - Plant Physiol. Bioch. 45: 162-167, 2007.

Ghasemi Y., Ghasemi K., Pirdashti H., Asgharzadeh R.: Effect of selenium enrichment on the growth, photosynthesis and mineral nutrition of broccoli. - Not. Sci. Biol. 8: 199-203, 2016.

Gohari G., Mohammadi A., Akbari A. et al:: Titanium dioxide nanoparticles $\left(\mathrm{TiO}_{2} \mathrm{NPs}\right)$ promote growth and ameliorate salinity stress effects on essential oil profile and biochemical attributes of Dracocephalum moldavica. - Sci. Rep.-UK 10: $912,2020$.

Gómez-Merino F.C., Trejo-Téllez L.I.: The role of beneficial elements in triggering adaptive responses to environmental stressors and improving plant performance. - In: Vats S. (ed.): Biotic and Abiotic Stress Tolerance in Plants. Pp. 137-172. Springer, Singapore 2018.

Habibi G.: Effect of drought stress and selenium spraying on photosynthesis and antioxidant activity of spring barley. Acta Agr. Slov. 101: 31-39, 2013.

Habibi G.: Selenium ameliorates salinity stress in Petroselinum crispum by modulation of photosynthesis and by reducing shoot Na accumulation. - Russ. J. Plant Physiol. 64: 368-374, 2017a.

Habibi G.: Physiological, photochemical and ionic responses of sunflower seedlings to exogenous selenium supply under salt stress. - Acta Physiol. Plant. 39: 213, 2017 b.

Haghighi M., Ramezani M.R., Rajaii N.: Improving oxidative damage, photosynthesis traits, growth and flower dropping of pepper under high temperature stress by selenium. - Mol. Biol. Rep. 46: 497-503, 2019.

Handa N., Kohli S.K., Sharma A. et al.: Selenium modulates dynamics of antioxidative defence expression, photosynthetic attributes and secondary metabolites to mitigate chromium toxicity in Brassica juncea L. plants. - Environ. Exp. Bot. 161: 180-192, 2019.

Hedrich R., Shabala S.: Stomata in a saline world. - Curr. Opin. Plant Biol. 46: 87-95, 2018.

Herrett R.A., Hatfield H.H., Crosby D.G., Vlitos A.J.: Leaf abscission induced by the iodide ion. - Plant Physiol. 37: 358363, 1962.

Hong F., Wang L., Meng X. et al.: The effect of cerium (III) on the chlorophyll formation in spinach. - Biol. Trace Elem. Res. 89: 263-276, 2002.

Hussain S., Iqbal N., Brestic M. et al.: Changes in morphology, chlorophyll fluorescence performance and Rubisco activity of soybean in response to foliar application of ionic titanium under normal light and shade environment. - Sci. Total Environ. 658: 626-637, 2019b.

Hussain S., Iqbal N., Raza M.A. et al.: Distribution and effects of ionic titanium application on energy partitioning and quantum yield of soybean under different light conditions. Photosynthetica 57: 572-580, 2019a.

Hussain S., Li S.X., Mumtaz M. et al.: Foliar application of silicon improves stem strength under low light stress by regulating lignin biosynthesis genes in soybean (Glycine max (L.) Merr.). - J. Hazard. Mater. 401: 123256, 2021.

Hussain T., Koyro H.-W., Zhang W. et al.: Low salinity improves photosynthetic performance in Panicum antidotale under drought stress. - Front. Plant Sci. 11: 481, 2020.

Izydorczyk G., Ligas B., Mikula K. et al:: Biofortification of edible plants with selenium and iodine - A systematic literature review. - Sci. Total Environ. 754: 141983, 2021.

Jiang C., Zu C., Lu D. et al.: Effect of exogenous selenium supply on photosynthesis, $\mathrm{Na}^{+}$accumulation and antioxidative capacity of maize (Zea mays L.) under salinity stress. - Sci. 
Rep.-UK 7: 42039, 2017.

Jiang C., Zu C., Shen J. et al.: Effects of selenium on the growth and photosynthetic characteristics of flue-cured tobacco (Nicotiana tabacum L.). - Acta Soc. Bot. Pol. 84: 71-77, 2015.

Kaur S., Kaur N., Siddique K.H.M., Nayyar H.: Beneficial elements for agricultural crops and their functional relevance in defence against stresses. - Arch. Agron. Soil Sci. 62: 905-920, 2016.

Kiferle C., Martinelli M., Salzano A.M. et al.: Evidences for a nutritional role of iodine in plants. - Front. Plant Sci. 12: 616868, 2021.

Landini M., Gonzali S., Perata P.: Iodine biofortification in tomato. - J. Plant Nutr. Soil Sc. 174: 480-486, 2011.

Li X.X., Yu B., Dong Y.Y. et al.: Lanthanum chloride enhances the photosynthetic characteristics and increases konjac glucomannan contents in Amorphophallus sinensis Belval. Photosynthetica 58: 165-173, 2020.

Liu X.Q., Huang H., Liu C. et al.: Physico-chemical property of rare earths. Effects on the energy regulation of photosystem II in Arabidopsis thaliana. - Biol. Trace Elem. Res. 130: 141151, 2009.

Liu X.Q., Su M.Y., Liu C. et al.: Effect of 4 f electron characteristics and alternation valence of rare earths on photosynthesis: Regulating distribution of energy and activities of spinach chloroplast. - J. Rare Earth. 25: 495-501, 2007a.

Liu X.Q., Su M.Y., Liu C. et al: Effects of $\mathrm{CeCl}_{3}$ on energy transfer and oxygen evolution in spinach photosystem II. J. Rare Earth. 25: 624-630, 2007b.

Luo H.W., He L.X., Du B. et al.: Foliar application of selenium (Se) at heading stage induces regulation of photosynthesis, yield formation, and quality characteristics in fragrant rice. Photosynthetica 57: 1007-1014, 2019.

Lyu S., Wei X., Chen J. et al.: Titanium as a beneficial element for crop production. - Front. Plant Sci. 8: 597, 2017.

Ma Q., Yue L.J., Zhang J.L. et al.: Sodium chloride improves photosynthesis and water status in the succulent xerophyte Zygophyllum xanthoxylum. - Tree Physiol. 32: 4-13, 2012.

Ma Y., Zhang P., Zhang Z. et al.: Origin of the different phytotoxicity and biotransformation of cerium and lanthanum oxide nanoparticles in cucumber. - Nanotoxicology 9: 262270, 2015.

Ma Y., Zou H., Gu H. et al.: Stimulatory effect of lanthanum nitrate on the root tuber yield of Pseudostellaria heterophylla via improved photosynthetic characteristics. - J. Rare Earth. 35: 610-620, 2017.

Maathuis F.J.M.: Sodium in plants: perception, signalling, and regulation of sodium fluxes. - J. Exp. Bot. 65: 849-858, 2014.

Maghsoudi K., Emam Y., Pessarakli M.: Effect of silicon on photosynthetic gas exchange, photosynthetic pigments, cell membrane stability and relative water content of different wheat cultivars under drought stress conditions. - J. Plant Nutr. 39: 1001-1015, 2016.

Malagoli M., Schiavon M., dall'Acqua S., Pilon-Smits E.A.H.: Effects of selenium biofortification on crop nutritional quality. - Front. Plant Sci. 6: 280, 2015.

Marschner P.: Marschner's mineral nutrition of higher plants. Pp. 651. Academic Press, Amsterdam 2012.

Martínez J.-P., Kinet J.-M., Bajji M., Lutts S.: NaCl alleviates polyethylene glycol-induced water stress in the halophyte species Atriplex halimus L. - J. Exp. Bot. 56: 2421-2431, 2005.

Mroczek-Zdyrska M., Strubińska J., Hanaka A.: Selenium improves physiological parameters and alleviates oxidative stress in shoots of lead-exposed Vicia faba L. minor plants grown under phosphorus-deficient conditions. - J. Plant Growth Regul. 36: 186-199, 2017.
Nawaz F., Ahmad R., Ashraf M.Y. et al.: Effect of selenium foliar spray on physiological and biochemical processes and chemical constituents of wheat under drought stress. Ecotox. Environ. Safe. 113: 191-200, 2015.

Naz F.S., Yusuf M., Khan T.A. et al.: Low level of selenium increases the efficacy of 24-epibrassinolide through altered physiological and biochemical traits of Brassica juncea plants. - Food Chem. 185: 441-448, 2015.

Nyitrai P., Bóka K., Gáspár L. et al:: Characterization of the stimulating effect of low-dose stressors in maize and bean seedlings. - J. Plant Physiol. 160: 1175-1183, 2003.

Ohnishi J., Flügge U.-I., Heldt H.W., Kanai R.: Involvement of $\mathrm{Na}^{+}$in active uptake of pyruvate in mesophyll chloroplasts of some $\mathrm{C}_{4}$ plants: $\mathrm{Na}^{+} /$pyruvate cotransport. - Plant Physiol. 94: 950-959, 1990.

Pereira L.F., Martins S.C.V., Aucique-Pérez C.E. et al.: Silicon alleviates mesophyll limitations of photosynthesis on rice leaves infected by Monographella albescens. - Theor. Exp. Plant Phys. 32: 163-174, 2020.

Pilon-Smits E.A.H.: Selenium in plants. - In: Lüttge U., Beyschlag W. (ed.): Progress in Botany. Pp. 93-107. Springer, Cham 2015.

Pilon-Smits E.A.H., Quinn C.F., Tapken W. et al.: Physiological functions of beneficial elements. - Curr. Opin. Plant Biol. 12: 267-274, 2009.

Proietti P., Nasini L., Del Buono D. et al.: Selenium protects olive (Olea europaea L.) from drought stress. - Sci. Hortic.Amsterdam 164: 165-171, 2013.

Raliya R., Biswas P., Tarafdar J.C.: $\mathrm{TiO}_{2}$ nanoparticle biosynthesis and its physiological effect on mung bean (Vigna radiata L.).Biotechnol. Rep. 5: 22-26, 2015.

Rasouli F., Kiani-Pouya A., Tahir A. et al: A comparative analysis of stomatal traits and photosynthetic responses in closely related halophytic and glycophytic species under saline conditions. - Environ. Exp. Bot. 181: 104300, 2021.

Raven J.A.: Cycling silicon - the role of accumulation in plants. New Phytol. 158: 419-421, 2003.

Redondo-Gómez S., Mateos-Naranjo E., Figueroa M.E., Davy A.J.: Salt stimulation of growth and photosynthesis in an extreme halophyte, Arthrocnemum macrostachyum. - Plant Biol. 12: 79-87, 2010.

Salgado O.G.G., Teodoro J.C., Alvarenga J.P. et al.: Cerium alleviates drought-induced stress in Phaseolus vulgaris. J. Rare Earth. 38: 324-331, 2020.

Sepehri A., Gharehbaghli N.: Selenium alleviate cadmium toxicity by improving nutrient uptake, antioxidative and photosynthetic responses of garlic. - Russ. J. Plant Physiol. 66: 152-159, 2019.

Servin A.D., Morales M.I., Castillo-Michel H. et al.: Synchrotron verification of $\mathrm{TiO}_{2}$ accumulation in cucumber fruit: A possible pathway of $\mathrm{TiO}_{2}$ nanoparticle transfer from soil into the food chain. - Environ. Sci. Technol. 47: 11592-11598, 2013.

Sheik M.A., Hameed A., Farooq T., Ibrahim M.: Evaluation of sodium silicate as antioxidant activator and growth enhancer in wheat. - Agrochimica 62: 113-125, 2018.

Smoleń S., Kowalska I., Sady W.: Assessment of biofortification with iodine and selenium of lettuce cultivated in the NFT hydroponic system. - Sci. Hortic.-Amsterdam 166: 9-16, 2014.

Song A., Li P., Fan F. et al.: The effect of silicon on photosynthesis and expression of its relevant genes in rice (Oryza sativa L.) under high-zinc stress. - PLoS ONE 9: e113782, 2014.

Tadina N., Germ M., Kreft I. et al.: Effects of water deficit and selenium on common buckwheat (Fagopyrum esculentum Moench.) plants. - Photosynthetica 45: 472-476, 2007. 
Tighe-Neira R., Reyes-Díaz M., Nunes-Nesi A. et al.: Titanium dioxide nanoparticles provoke transient increase in photosynthetic performance and differential response in antioxidant system in Raphanus sativus L. - Sci. Hortic.Amsterdam 269: 109418, 2020.

Tripathi P., Na C.-I., Kim Y.: Effect of silicon fertilizer treatment on nodule formation and yield in soybean (Glycine max L.). Eur. J. Agron. 122: 126172, 2021.

Trippe R.C., Pilon-Smits E.A.H.: Selenium transport and metabolism in plants: Phytoremediation and biofortification implications. - J. Hazard. Mater. 404: 124178, 2021.

Vatansever R., Ozyigit I.I., Filiz E.: Essential and beneficial trace elements in plants, and their transport in roots: a review. Appl. Biochem. Biotech. 181: 464-482, 2017.

Verma K.K., Wu K.-C., Verma C.L. et al.: Developing mathematical model for diurnal dynamics of photosynthesis in Saccharum officinarum responsive to different irrigation and silicon application. - PeerJ 8: e10154, 2020.

White P.J.: Selenium metabolism in plants. - BBA-Gen. Subjects 1862: 2333-2342, 2018.

Wu H., Tito N., Giraldo J.P.: Anionic cerium oxide nanoparticles protect plant photosynthesis from abiotic stress by scavenging reactive oxygen species. - ACS Nano 11: 11283-11297, 2017.

Xue T., Hartikainen H., Piironen V.: Antioxidative and growthpromoting effect of selenium on senescing lettuce. - Plant
Soil 237: 55-61, 2001.

Yamamoto T., Nakamura A., Iwai H. et al.: Effect of silicon deficiency on secondary cell wall synthesis in rice leaf. J. Plant Res. 125: 771-779, 2012.

Yildiztugay E., Ozfidan-Konakci C., Kucukoduk M., Tekis S.A.: The impact of selenium application on enzymatic and nonenzymatic antioxidant systems in Zea mays roots treated with combined osmotic and heat stress. - Arch. Agron. Soil Sci. 63: 261-275, 2017.

Ze Y., Liu C., Wang L. et al.: The regulation of $\mathrm{TiO}_{2}$ nanoparticles on the expression of light-harvesting complex II and photosynthesis of chloroplasts of Arabidopsis thaliana. Biol. Trace Elem. Res. 143: 1131-1141, 2011.

Zhang J., Zou W., Li Y. et al.: Silica distinctively affects cell wall features and lignocellulosic saccharification with large enhancement on biomass production in rice. - Plant Sci. 239: 84-91, 2015.

Zhang M., Tang S., Huang X. et al.: Selenium uptake, dynamic changes in selenium content and its influence on photosynthesis and chlorophyll fluorescence in rice (Oryza sativa L.). - Environ. Exp. Bot. 107: 39-45, 2014.

Zhang Z., He X., Zhang H. et al.: Uptake and distribution of ceria nanoparticles in cucumber plants. - Metallomics 3: 816-822, 2011.

(C) The authors. This is an open access article distributed under the terms of the Creative Commons BY-NC-ND Licence. 